\title{
Study on Floating Attenuator Wave Energy Devices
}

\author{
by Brian M. Count* Takeaki Miyazaki**, Member
}

\begin{abstract}
Summary
In this paper the theory of optimal control is developed in a form in which non absorbing modes of motion may be included in any system. The theory is developed for wave energy absorption by phase controlled systems and ones where only a simple resistive damping may be applied.

The theory is applied to two attenuators based on the Kaimei (an attenuator) concept. Firstly, a system of vertically oscillating pistons are considered which is intended to model a device with bottom opening oscillating water columns, and secondly horizontally moving pistons are considered. The latter system is analogous to a device containing side opening oscillating water columns.

Results are shown for both devices when constrained so that only the column motions are possible and for the case where the systems float freely. In addition, power extraction systems corresponding to phase control and resistive damping alone are considered. The conclusions from the study are unexpected in that floating attenuators perform better than fixed ones. Less surprising is the fact that phase controlled systems are considerably more effective than simple damped ones. Overall the results supported a bottom opening floating attenuator to be superior to other configurations although to what extent this conclusion is dependent upon the actual device geometries chosen is difficult to deduce.
\end{abstract}

\section{Introduction}

It has recently been announced in Japan that 400 Million yen (about $£ 1 \mathrm{~m}$ is to be provided over the next four years in order that further sea trials may be undertaken with the Kaimei device (Masuda, et al., 1980) ${ }^{1}$ ). This funding, it should be noted, does not make any provision for effort in the Japanese wave power programme, which will be provided by a separate budget. Therefore this project, based at the Japan Marine Science and Technology Centre, will be the largest activity in the wave energy field and it will be important to assess its potential.

The Japanese studies will continue to be based on the Kaimei device which was tested at large scale between 1977-1980. Other countries, namely the UK, USA, Canada and Ireland, were also involved in this project through the auspices of the International Energy Agency in Paris. Unfortunately, the results from the Kaimei tests

* Marchwood Engineering Laboratories, Central Electricity Generating Board, U.K.

** Japan Marine Science and Technology Centre were generally disappointing in that the performance of the device was very much lower than hoped and in its present form would produce electricity at a very high cost.

However, Masuda and his fellow workers believe that the performance can be improved and eventually the floating attenuator (devices that are aligned parallel to the wave propagation direction) configurations will offer the most cost effective solution to wave energy. This is at variance to many of the UK and Norwegian studies where terminators (devices aligned perpendicular to the wave direction) figure prominently. Such differences in opinion are difficult to resolve and there is generally no simple answer but it is possible to make some predictions about the hydrodynamic efficiencies that could be achieved with an attenuator device. This may help to clarify the improvements that are available to Kaimei and act as some form of comparison with terminator systems.

The study described in this paper will be based upon linear hydro-dynamic theory that has been well validated in the wave power field (Count $1982)^{2}$. By using such techniques the wave forces on any floating structure can be determined and 
these are used to calculate the motion of the device when subject to some external damping system. As a result the absorbed power can be determined and this is maximised subject to various forms of possible power conversion systems. In this way upper limits to the absorption capability can be calculated and it will be shown that these figures are consistent with experimental measurements.

\section{The Theory}

Consider only wave power device which has $\mathrm{n}$ independent degrees of freedom. This can be described by an equation of motion of the form

$$
\boldsymbol{I} \ddot{\boldsymbol{x}}+\boldsymbol{B} x=f^{\boldsymbol{W}}(t)+f^{\mathrm{ex} \mathrm{t}}(t)
$$

where $\boldsymbol{I}$ is the inertia matrix of the dry body, $\boldsymbol{B}$ the linearised hydrostatic matrix, $f^{W}(t)$ is a vector of the wave induced forces, $f^{\text {ext }}(t)$ the externally applied force and $x$ is a vector of displacements. In general the wave forces are expressed (in linear hydrodynamic theory) as:

$$
f^{W}(t)=f_{0}(t)-\boldsymbol{M}(\omega) \ddot{x}-\boldsymbol{N}(\omega) \dot{x}
$$

where $f_{0}(t)$ is the wave induced force on a stationary device with $\boldsymbol{M}(\omega)$ and $\boldsymbol{N}(\omega)$ as the added mass and damping matrices respectively whose elements are frequency dependent. Strictly this representation is only valid for steady harmonic oscillations at an angular frequency $\omega$ since all calculations will be carried out with a linear external force and monochromatic incident waves this condition is satisfied. This is, of course, equivalent to a Fourier analysis of irregular wave responses of a linear system.

\subsection{Optimal Control}

Rearranging equation (2.1) and considering the case where

$$
\begin{aligned}
& x(t)=X(\omega) \mathrm{e}^{-\mathrm{i} \omega t} \\
& f_{0}(t)=F_{0}(\omega) \mathrm{e}^{-\mathrm{i} \omega t}
\end{aligned}
$$

and

$$
f^{\mathrm{ext}}(t)=-\boldsymbol{R} \boldsymbol{C} \boldsymbol{R}^{T} X \mathrm{e}^{-\mathrm{i} \omega t}
$$

$T$ denoting the matrix transpose. This gives an equation for $X$ of the form

$$
\left[\boldsymbol{G}+\boldsymbol{R} \boldsymbol{C} \boldsymbol{R}^{T}\right] X=F_{0}
$$

where

$$
\boldsymbol{G}=\boldsymbol{B}-\omega^{2}[\boldsymbol{I}+\boldsymbol{M}(\omega)]-\mathrm{i} \omega \boldsymbol{N}(\omega)
$$

The form of $f^{\mathrm{ext}}(t)$ is chosen so that only $m$ modes of motion are controlled $(m \leq n)$ and the matrix $\boldsymbol{R}$ is an $(n \times m)$ matrix whose columns are unit vectors corresponding to each mode of motion that is under control. The control matrix $\boldsymbol{C}$ is to be determined and the reason for introducing $\boldsymbol{R}$ was to enable an $(n \times n)$ matrix to be derived from $C$.
In this case the time averaged power absorbed is:

$$
\frac{1}{2} \omega \operatorname{Re}\left[\mathrm{i} \bar{X}^{T} \boldsymbol{R C} \boldsymbol{R}^{T} X\right]
$$

where the overbar denotes the complex conjugate.

Substituting for $X$ from equation (2.6) the time averaged power becomes,

$$
\begin{aligned}
\frac{\mathrm{i}}{4} \omega A^{2} \bar{F}_{0} \boldsymbol{T}\left[\left[\overline{\boldsymbol{G}}+\boldsymbol{R} \overline{\boldsymbol{C}} \boldsymbol{R}^{T}\right]^{T}\right]^{-1} \boldsymbol{R}(\boldsymbol{C}-\overline{\boldsymbol{C}}) \boldsymbol{R}^{T} \\
\quad \cdot\left[\boldsymbol{G}+\boldsymbol{R} \boldsymbol{C} \boldsymbol{R}^{T}\right]^{-1} F_{0}
\end{aligned}
$$

and maximising this expression with respect to the elements of the matrix $\boldsymbol{C}$ gives a condition,

$$
\boldsymbol{R} \boldsymbol{I}_{m} \boldsymbol{R}^{T}=\boldsymbol{R}\left(\boldsymbol{C}-\overline{\boldsymbol{C}}^{T}\right) \boldsymbol{R}^{T}\left(\boldsymbol{G}+\boldsymbol{R} \boldsymbol{C} \boldsymbol{R}^{T}\right)^{-1} \boldsymbol{R} \boldsymbol{I}_{m} \boldsymbol{R}^{T}
$$

where $\boldsymbol{I}_{m}$ is the $(m \times m)$ unit matrix. To solve this equation for $\boldsymbol{C}$, it is convenient to partition the $(n \times n)$ matrices so that in matrix form the above condition becomes,

$$
\left(\begin{array}{cc}
0 & 0 \\
0 & \boldsymbol{I}_{m}
\end{array}\right)=\left(\begin{array}{cc}
0 & 0 \\
0 & \boldsymbol{C}-\overline{\boldsymbol{C}}^{T}
\end{array}\right)\left(\begin{array}{cc}
\boldsymbol{\alpha} & \boldsymbol{\beta} \\
\boldsymbol{\gamma} & \boldsymbol{\delta}
\end{array}\right)\left(\begin{array}{cc}
0 & 0 \\
0 & \boldsymbol{I}_{m}
\end{array}\right)
$$

as long as the last $m$ modes are chosen to be controlled and $\boldsymbol{\alpha}, \boldsymbol{\beta}, \boldsymbol{\gamma}$ and $\boldsymbol{\delta}$ are the partition elements of $\left(\boldsymbol{G}+\boldsymbol{R} \boldsymbol{C} \boldsymbol{R}^{T}\right)^{-1}$. This gives the relation

$$
\boldsymbol{I}_{m}=\left(\boldsymbol{C}-\overline{\boldsymbol{C}}^{T}\right) \boldsymbol{\delta}
$$

and it can be shown by simple algebra that

$$
\boldsymbol{\delta}^{-1}=\left[\boldsymbol{R}^{T} \boldsymbol{G}^{-1} \boldsymbol{R}\right]^{-1}+\boldsymbol{C}
$$

giving the optimal controller as

$$
\boldsymbol{C}=-\left[\left[\boldsymbol{R}^{T} \overline{\boldsymbol{G}}^{-1} \boldsymbol{R}\right]^{T}\right]^{-1}
$$

It should be noted that this result assumes that $\boldsymbol{\delta}^{-1}$ exists and in some cases this is not true.

\subsection{Constrained Linear Control}

The above analysis derives the optimal power extraction characteristics for any particular wave energy device. Implicit in this derivation is that the resultant amplitudes and phases of the motion are unconstrained and in many cases the amplitudes predicted are excessively large. Therefore, it would be more realistic if the motion of the absorber is limited in some manner. Evans $(1981)^{3)}$ has suggested a convenient global condition that

$$
\left|\boldsymbol{R}^{T} X\right| \leq m \beta
$$

where $\beta$ is some prescribed value, typically the same order as the incident wave amplitude. If the solution to the previous equation satisfies this condition then the optimal controller is given by equation (2.14). However, in the case where this solution would give rise to movements that exceed the criterion (2.15) then it is possible to modify the optimal controller such that the oscillating solution which satisfies

$$
\left|\boldsymbol{R}^{T} X\right|=m \beta
$$


is chosen, (Evans 1981) ${ }^{3}$.

This may not necessarily give the most satisfactory solution to constrained movement since the above approach firstly uses a constraint on the summed displacements rather than ensuring that all displacements are individually limited, and secondly a linear solution has been assumed whereas the motion should only be limited during periods when the movement exceeds it's limit. However, the solutions from this approach are likely to be indicative of the behaviour under limited movement and the alternative is a difficult task to undertake.

Therefore, taking the constraint as,

$$
\bar{X}^{T} \boldsymbol{R} \boldsymbol{R}^{T} X=(m \beta)^{2}
$$

a similar analysis to Section 2.1 will be carried out with the additional term

$$
\omega^{2} \mu\left[\bar{X}^{T} \boldsymbol{R} \boldsymbol{R}^{T} X-(m \beta)^{2}\right] / 2
$$

in the optimisation function where $\omega^{2} \mu / 2$ is a real Lagrange multiplier. Maximising expression (2.8) plus (2.17) with respect to the matrix $\boldsymbol{C}$, gives the equation

$$
\begin{aligned}
\boldsymbol{R} \boldsymbol{I}_{m} \boldsymbol{R}^{T}= & \boldsymbol{R}\left(\boldsymbol{C}-\overline{\boldsymbol{C}}^{T}-2 \mathrm{i} \omega \mu \boldsymbol{I}_{m}\right) \boldsymbol{R}^{T} \\
& \cdot\left(\boldsymbol{G}+\boldsymbol{R} \boldsymbol{C} \boldsymbol{R}^{T}\right)^{-1} \boldsymbol{R} \boldsymbol{I}_{m} \boldsymbol{R}^{T}
\end{aligned}
$$

which reduces to

$$
\boldsymbol{C}=-\left[\left[\boldsymbol{R}^{T} \overline{\boldsymbol{G}}^{-1} \boldsymbol{R}\right]^{T}\right]^{-1}-2 \mathrm{i} \omega \boldsymbol{\mu} \boldsymbol{I}_{m}
$$

and therefore the parameter $\mu$ can be determined from equation (2.17) by substituting for $\boldsymbol{C}$ in the expression for $X$ (equation 2.6).

It is interesting to note that the effect of constraining the motion is to introduce an equal damping factor of $2 \mu$ into each mode of controllable motion. This has the effect of reducing the motion amplitude to the desired values.

However, it is important to note that in both of the above derivations the control matrix $\boldsymbol{C}$ has adjusted the phase of the motion to allow maximum energy capture from the waves. This implies, in this linear formulation, that reactive components are available to store and release energy over the wave cycle. The only practical realisation of such a system is that of phase control proposed by Budal and Falnes (1982) ${ }^{4}$ and although their scheme is nonlinear in character the above analysis will determine the bounds of possibility.

Unfortunately, the phase control concept is by no means fully developed and most current designs of wave energy devices use a simple resistive turbine for power extraction. It is therefore partinent to calculate the optimal performance of a system that is allowed to extract energy in the controllable modes through a simple resistive damper alone.

\subsection{Simple Resistive Power Extraction}

Whilst it would be desirable, in terms of energy generation, to use turbines of different characteristics on each absorbing element (mode) of the device, in practice it is very likely that identical machines would be deployed. In addition, the latter condition proves a more tractable problem to solve

$$
f^{\mathrm{ext}}(t)=\mathrm{i} \omega D \boldsymbol{R} \boldsymbol{I}_{m} \boldsymbol{R}^{\boldsymbol{T}} X \mathrm{e}^{-\mathrm{i} \omega t}
$$

which corresponds to equal damping applied to the $m$ absorbing modes of motion with $\boldsymbol{R}$ and $X$ as previously defined. As before the time averaged power is

$$
\frac{1}{2} \omega R \mathrm{e}\left[\mathrm{i} D \bar{X}^{T} \boldsymbol{R} \boldsymbol{I}_{m} \boldsymbol{R}^{T} X\right]
$$

and this expression is to be maximised with respect to the scalar parameter $D$. Differentiating the above expression with respect to $D$ and rearranging gives an expression for $D$ as the solution of

$$
\begin{aligned}
& \bar{X}^{T}\left[\mathrm{i} D \boldsymbol{I}_{m}\left\{\boldsymbol{R} \boldsymbol{I}_{m} \boldsymbol{R}^{T}\left(\boldsymbol{Z}-\overline{\boldsymbol{Z}}^{-1}\right) \boldsymbol{R} \boldsymbol{I}_{m} \boldsymbol{R}^{T}\right\}\right. \\
& \left.\quad+\boldsymbol{R} \boldsymbol{I}_{m} \boldsymbol{R}^{T}\right] X=0
\end{aligned}
$$

where

$$
X=Z^{-1} F_{0} \text { and } \boldsymbol{Z}=\boldsymbol{G}-\mathrm{i} \omega D \boldsymbol{R} \boldsymbol{I}_{m} \boldsymbol{R}^{T}
$$

with $\boldsymbol{G}$ and $F_{0}$ as defined in Section 2.1.

In addition, the case of constrained optimisation must be considered where

$$
\bar{X}^{T} \boldsymbol{R} \boldsymbol{R} X \leq(m \beta)^{2}
$$

and if the optimal condition determined above violates this imposed motion constraint then, as was shown in Section 2.2, an additional increment of damping must be applied. Therefore, in this case the optimum damping with constraints is given by the equation

$$
\bar{X}^{T} \boldsymbol{R}^{T} X=(m \beta)^{2}
$$

\section{Application of the Theory}

The theory described in the previous section can now be used to calculate the performance of any wave energy device provided the appropriate hydrodynamic parameters can be computed. Fortunately, this can be achieved by using numerical techniques based on boundary integral value methods (Count, 1982) ${ }^{2}$.

\subsection{A Bottom Opening Attenuator Model}

The first configuration studied was a bottom opening set of oscillating water columns as shown in Figure 1. The buoyancy chambers were chosen on the basis of previous experimental studies on Kaimei (Miyazaki et al. 19785); Fry and Jefferys $1979^{6)}$ ) that demonstrated most power was absorbed from the front, middle and rear chambers of the device. This effect, which has been attributed to the pitching action of the device, has 
Plan

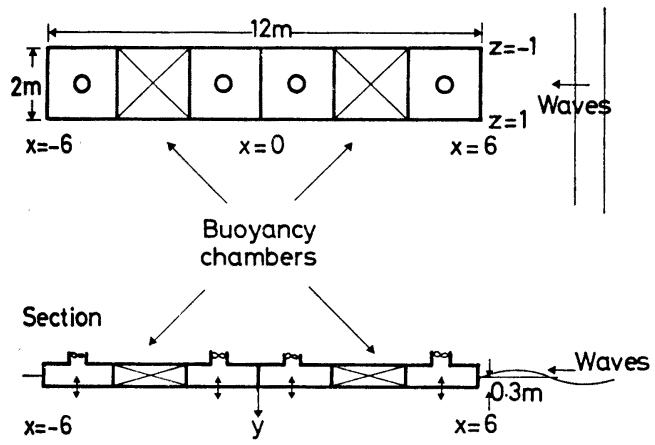

Fig. 1 The configuration of a bottom opening attenuator model
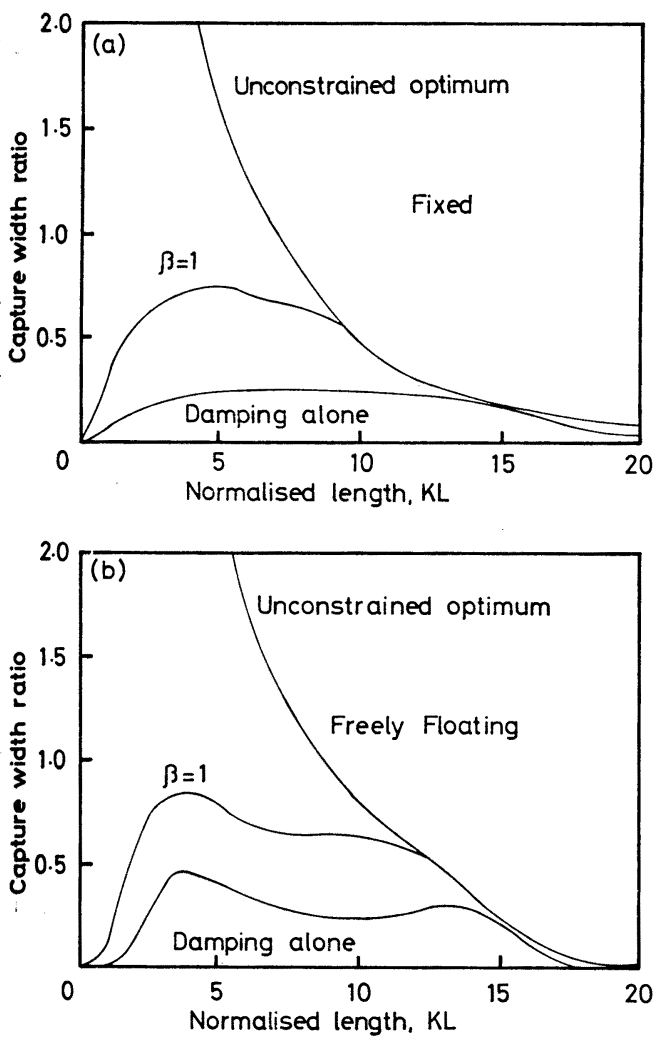

Fig. 2 The performance of a bottom opening attenuator both fixed and freely floating

determined the choice of the buoyancy compartments. Naturally, by choosing a specific chamber configuration the study cannot be considered as exhaustive but it is likely that performance calculations will be indicative of attenuator behaviour.

In Figure 2 the performance of the device is given for both the cases of optimal control (phase control say) and with resistive damping alone. It should be noted that in the latter case the damping constant is optimised at each frequency. In Figure $2 \mathrm{a}$ the curves are for a fixed device so that only motion within the chambers is excited by the wave. For Figure $2 b$ the complete structure is free to move like any ship.

In computing the results various assumptions have to be made. Firstly, the structure is assumed to be rigid with the water columns moving as pistons whose masses are exactly that of the entrained water. Secondly, in order that the freely floating case may be tackled it has been assumed that the structure has a uniform mass distribution and its density is one half that of water. This implies that there would be an equal amount of structure above the water line which is clearly not the case from a naval architecture viewpoint. However, this does allow easy computation of the inertia and spring matrices.

These results (Figure 2) indicate some interesting features. Firstly, the curves for the fixed system show that a constrained motion $(\beta=1)$ with phase control can give a high absorption capability. (N.B. the capture width ratio is the power absorbed divided by the incident wave power in a frontage $L$ ). However, for damping alone this absorption is considerably reduced and whilst the actual shape is dependent upon the inertia and spring characteristics of the columns, it does demonstrate that hydrodynamic interactions between chambers are important. If this were not the case and the matrices were diagonal then a peak value, corresponding to the resonant frequency, should lie on the phase controlled curve. However, this is not the case.

Secondly, and perhaps more surprising, are the results for a freely floating structure which show an improvement over the fixed system. It was anticipated that non-absorbing rigid body motions (heave, sway, surge, pitch, roll and yaw) would reduce performance but clearly there is some advantageous coupling between the chamber motions and these rigid ones. To what extent this is a function of the particular structure chosen for the study is not known but it does demonstrate an unexpected characteristic.

To investigate the form of this coupling between the rigid body and chamber motions calculations are shown in Figure 3 where the heaving and pitching motions of the device are separately restrained. The results are for head seas (see Figure 1) and apply to a system that can absorb energy only from a resistive damping. In Figure $3 a$ results for a freely floating and fixed device are compared with those for a system whose heave (vertical) movement is forced to be zero. Simi- 
larly in Figure $3 \mathrm{~b}$ the freely floating and fixed results are compared with those where the pitch motion (rotation) is completely eliminated. From the curves it can be seen that by suppressing the heave motion the higher frequency $(\mathrm{KL} \simeq 13)$ peak is eliminated whereas the lower frequency
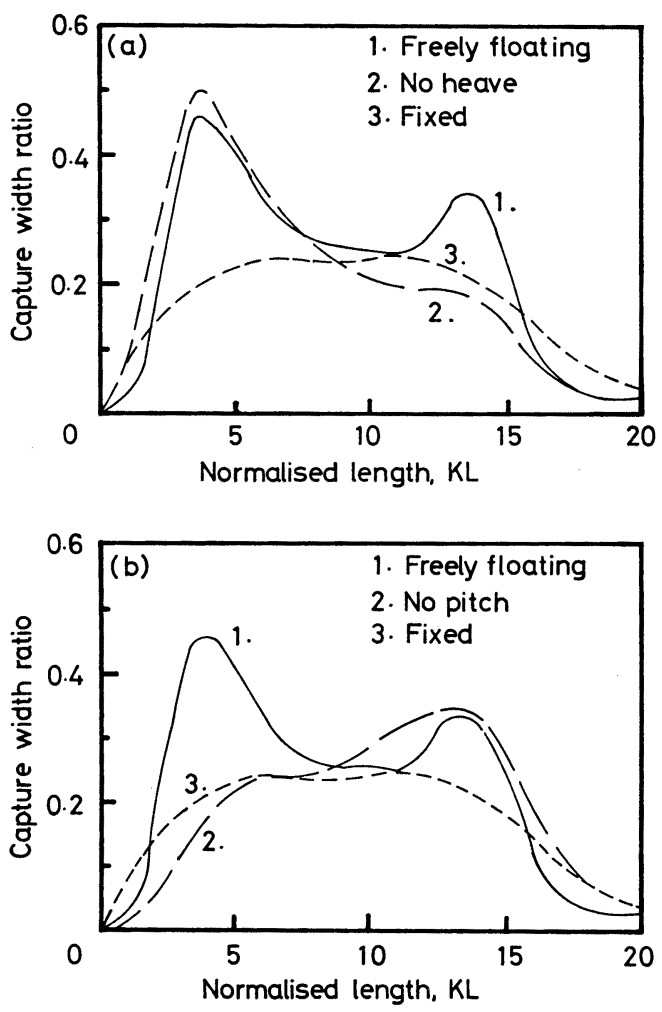

Fig. 3 The performance of a bottom opening device with restraints on heave and pitch motions

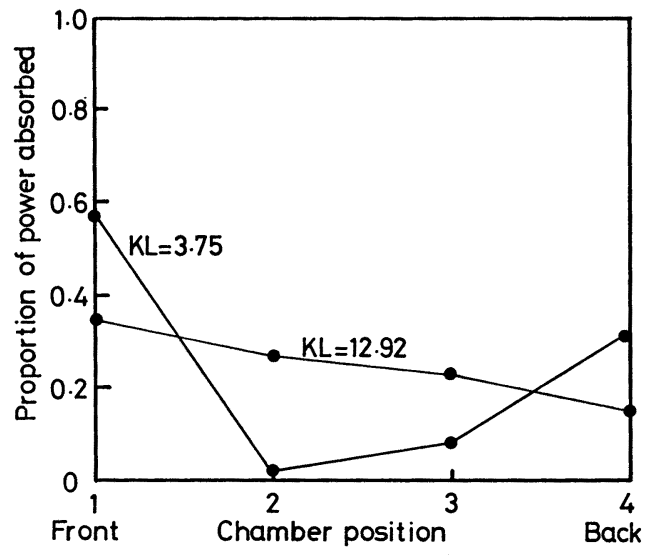

Fig. 4 The distribution of power absorption along the device length
( $K L \simeq 4)$ peak is dependent upon the pitching motion. This is supported by Figure 4 where the distribution of energy absorption is shown for the freely floating case at the higher and lower frequency performance peaks. The results demonstrate that for $\mathrm{KL}=3.75$ the front (chamber position 1 ) and back chambers absorb most energy, consistent with a pitching effect, whereas for $\mathrm{KL}=12.92$ the distribution is more even with some attenuation from front to back.

As a result of these calculations it would appear that the bottom opening device chosen for this study has a performance that is advantageously assisted by pitching for long wavelengths (low frequencies) and heaving for medium wavelengths. It should be noted that surge (horizontal) motion has little or no effect on device performance due to the shallow draught of the structure and hence a relatively weak hydrodynamic coupling with the chamber movements.

\subsection{A Side Opening Attenuator Model}

The second configuration studied is shown in Figure 5 and consists of oscillating water columns that have vertical side openings to the sea. The buoyancy chambers are positioned in a similar manner to the previous, bottom opening, system.

Performance tests are shown in Figures 6 for a fixed (Figure 6a) and freely floating (Figure 6b) device both with and without phase control. In the case of a freely floating device with damping alone however, there is little to choose between the peak performances but since the side opening system has its peak at a higher frequency (larger $\mathrm{KL}$ ) it would not appear so attractive.

In Figures $7 \mathrm{a}$ and $7 \mathrm{~b}$ the performance is shown
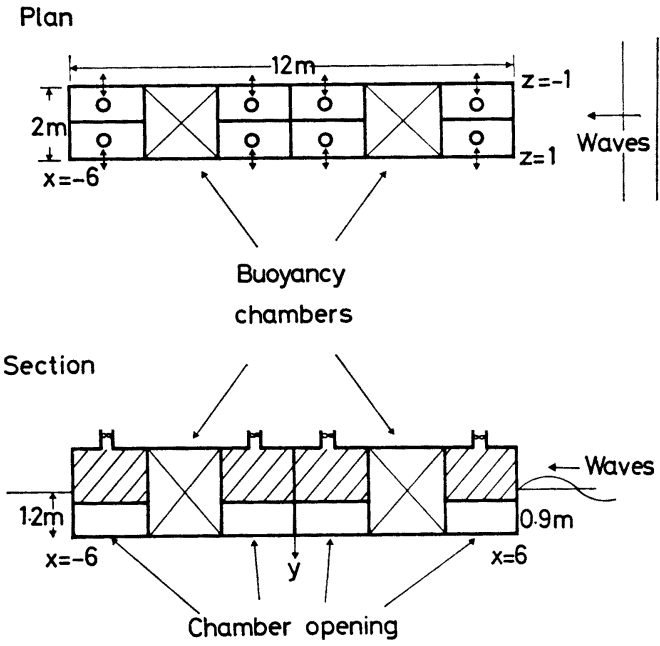

Fig. 5 The configuration of a side opening attenuator with 4 pairs of absorbing chambers 

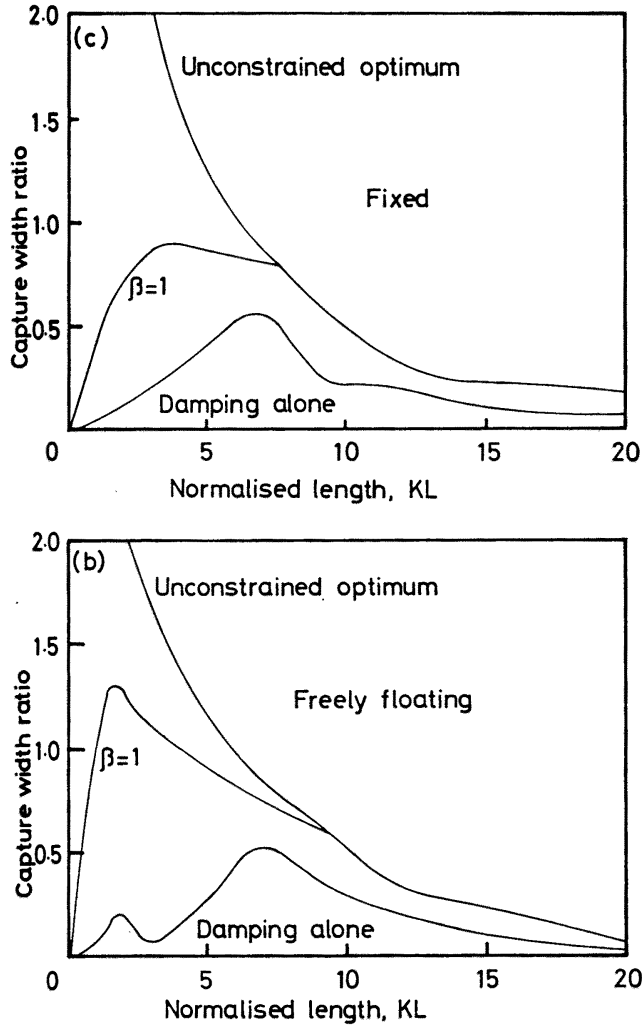

Fig. 6 The performance of a side opening attenuator both fixed and freely floating

subject to various restraints of the rigid body motions for the side opening device absorbing energy through resistive damping alone. In this case only minor modifications are introduced by suppressing heave or pitch motions showing that this device does not have such strong coupling between the chamber and the rigid body motions. This weak coupling may have been anticipated since the incident wave direction is orthogonal to the chamber motions. As a result, the distribution of absorbed energy along the length of the device, shown in Figure 8, has no distinctive pattern.

\section{Experimental Correlation}

\subsection{A Bottom Opening Device}

To test the validity of the theory it is interesting to compare the above predictions with available experimental data. For the bottom opening system data has been taken from Kinoshita et al. $(1982)^{7)}$ who published experimental results for a device with 16 bottom opening chambers and buoyancy compartments arranged along the length of the device (on both sides). This is
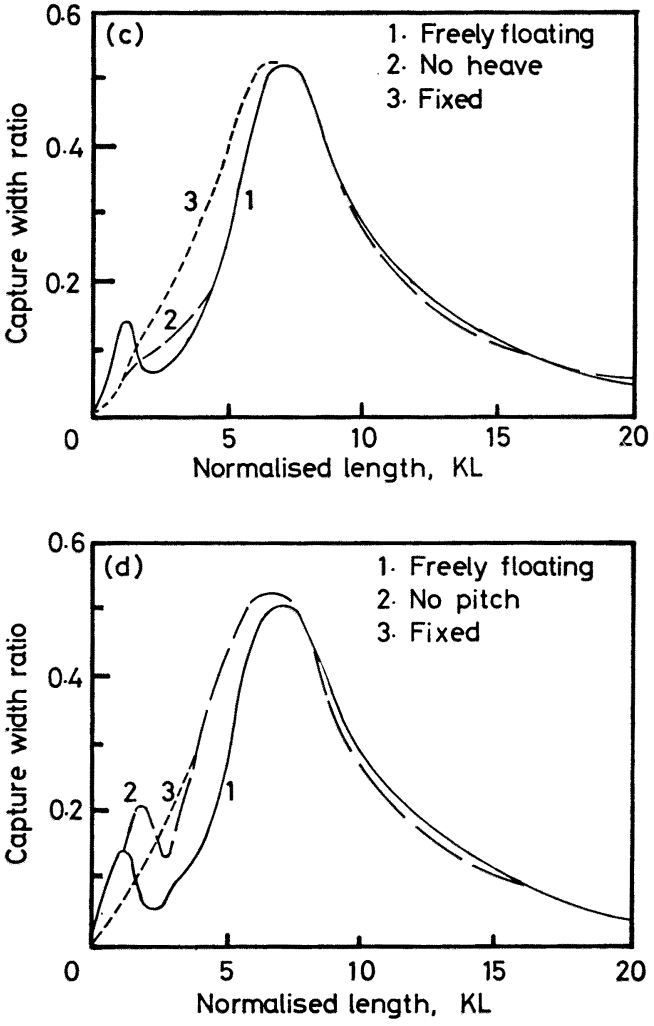

Fig. 7 The performance of a side opening device with restraints on heave and pitch motions

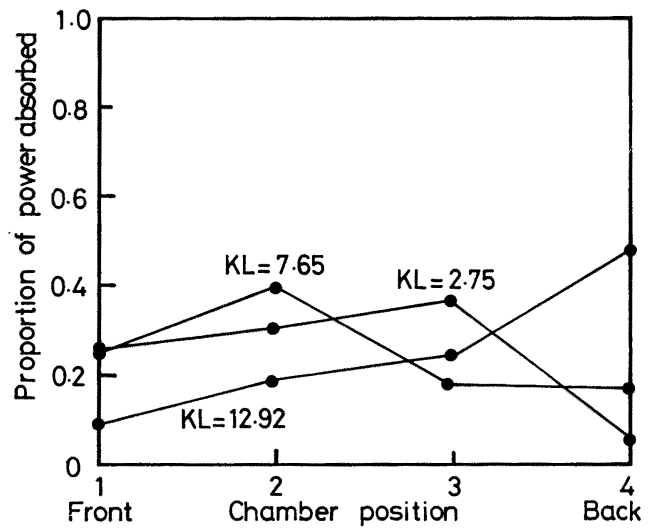

Fig. 8 The distribution of power absorption along the device length

known as the catamaran device.

The experimental results are shown in Figure 9 for this device when tested in regular waves with a single, constant, resistive absorber on each chamber in the form of an 'orifice' assembly. 


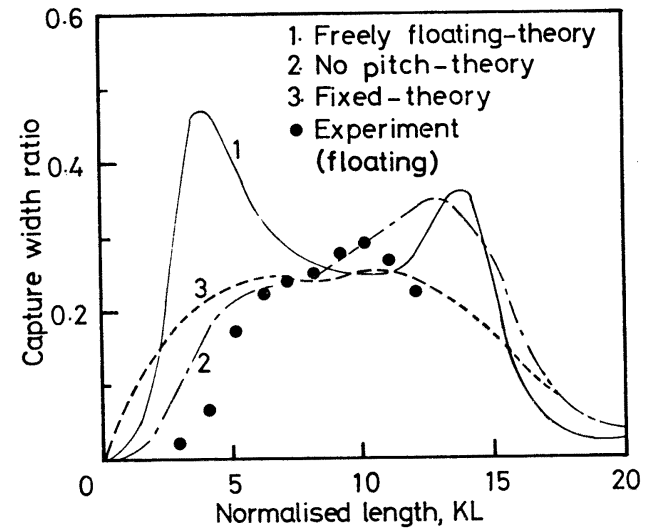

Fig. 9 Comparison between theory and experiment for a bottom opening attenuator

Also shown on the figure are theoretical results for the optimal performance of a system assuming that the resistance can be changed at each frequency.

It can be seen that the correlation between the experiments and theory for a freely floating device is poor. However, this is not surprising since it has been shown earlier in this paper that there is important coupling between the chamber and rigid body motions. As a result it would be anticipated that the device dynamics should be correctly represented in order to obtain good correlation. Unfortunately, this information is not available.

It is interesting to speculate on the reasons for the discrepancy and shown in the figure are also the theoretical calculations assuming that the device is restrained from pitching and held fixed. These curves fit the experimental data much better and, bearing in mind that the experimental values do not correspond to an optimised damping parameter, this would indicate that the pitch and heave induced peaks are not obtained in practice. Whether this is due to the device dynamics or the mooring arrangement is open to question.

\subsection{A Side Opening System}

Fortunately, since this system is less influenced by the rigid body movements and, moreover, experimental data corresponding to optimised damping at such frequency is available, it is possible to test the theory with comparable model test results. Miyazaki et al. $(1982)^{8}$ ) have published data for tests on a 32 chamber model (16 symmetrically placed pairs) which absorbed energy via orifice plates. These tests also investigated the variation of performance, at each

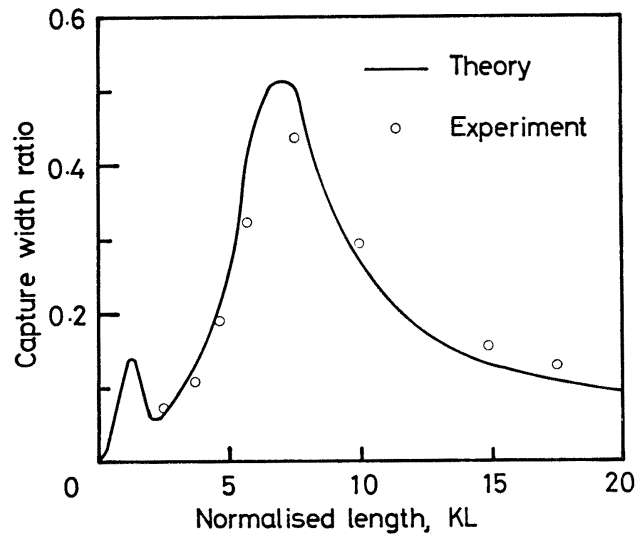

Fig. 10 Comparison between theory and experiment for a side opening attenuator

frequency, with orifice damping and therefore it was possible to select an optimum performance characteristic.

The results are shown in Figure 10 where the theory is for a freely floating system. It is encouraging to observe the close correlation with the test data and it would seem to indicate that a few side opening chambers are sufficient to model the hydrodynamic character of such a device. These results clearly add confidence to the theory.

\section{Conclusions}

This study cannot be considered as exhaustive but some interesting points have emerged as a result of the limited number of calculations.

Firstly, according to the theory, if resistive damping alone is available for power extraction a floating bottom opening system appears most attractive. This assumes that the shallow draught structure is able to utilise the pitch behaviour to give improved absorption at long wavelengths. However, the experimental measurements (Figure 9) did not confirm this effect and its sensitivity to structural design and mooring arrangement may prove to be a problem.

In any case the maximum performance that can be anticipated from a floating attenuator without the use of phase control is a capture width ratio of 0.5 . This is in contrast to a fixed terminator (a device aligned perpendicular to the wave direction) which can achieve values of 1.0 , or greater, although at higher capital costs.

Finally, as expected the introduction of phase control considerably improves the performance. It appears, therefore, that the development of this concept is essential if attenuator devices are 
to have any chance of producing wave energy economically.

\section{Acknowledgement}

This paper is published by permission of the Central Electricity Generating Board.

\section{References}

1) Masuda, et al.: The sea trials and discussions on the wave power generator ship 'Kaimei', Proc. of 13th Symp. on Naval Hydrodynamics, published by Shipbuilding Research Association of Japan, 1980.

2) Count, B. M.: On the physics of absobing energy from ocean waves, $\mathrm{Ph}$. $\mathrm{D}$. Thesis, Dept. of Physics, University of Exeter, 1982.

3) Evans, D. V.: Maximum wave power absorption under motion constraints, Appl. Ocean Research, 3, pp. 200-203, 1981.

4) Budal and Falnes: The Norwegian Wave Power Buoy Project, The 2nd International
Symposium on Wave Energy Utilization Norway, 1982.

5) Miyazaki, T. and Masuda, Y.: Research and Development on Wave Power Electricity" Generation System, Oceanology International '78, 1978.

6) Fry, R. and Jefferys, E. R.: Tank trials of a model Kaimei, CEGB Laboratory Note No. R/M/N1072, 1979.

7) Kinoshita, T., Saijo, K. and Yokomizo, H.: Theoretical analysis of performance of Kaimei for wave power absorber, $J$ of Kansai Soc. of Naval Arch. of Japan, (In Japanese) 1982.

8) Miyazaki, T., Yokomizo, H. Washio, Y. and Tsuzuku, T.: Model test results of Kaimei with bottom plate, Paper $\mathrm{V}$ of the wave power generator Kaimei research report No. 2 pub by JAMSTEC (In Japanese), 1982. 